CONFORMAL GEOMETRY AND DYNAMICS

An Electronic Journal of the American Mathematical Society

Volume 7 , Pages $34-48$ (June 17, 2003)

S $1088-4173(03) 00081-\mathrm{X}$

\title{
VOLUMES OF HYPERBOLIC 3-MANIFOLDS. NOTES ON A PAPER OF GABAI, MEYERHOFF, AND MILLEY
}

\author{
T. H. MARSHALL AND G. J. MARTIN
}

\begin{abstract}
We present a new approach and improvements to the recent results of Gabai, Meyerhoff and Milley concerning tubes and short geodesics in hyperbolic 3-manifolds. We establish the following two facts: if a hyperbolic 3 -manifold admits an embedded tubular neighbourhood of radius $r_{0}>1.32$ about any closed geodesic, then its volume exceeds that of the Weeks manifold. If the shortest geodesic of $M$ has length less than $\ell_{0}<0.1$, then its volume also exceeds that of the Weeks manifold.
\end{abstract}

\section{INTRODUCTION}

In the paper [6], Gabai, Meyerhoff and Milley establish the compactness of a certain parameter space which they propose to search, following their earlier work with Thurston [7, to identify the smallest volume hyperbolic 3-manifold. They also discuss the feasibility of this search and other computational issues. In this note we introduce a new approach for investigating these spaces. We prove that 0.2855 is a lower bound for the volume of any hyperbolic $3-$ manifold. (The previous best known bound was 0.27 obtained by Przeworski [16, following [10. Recently, Algol has improved this to 0.32 using entirely different techniques.) The conjectural sharp value here is that of the Weeks manifold with a volume of about 0.9427 . We establish the following two facts: if a hyperbolic 3-manifold admits an embedded tubular neighbourhood of radius $r_{0}>1.31$ about any closed geodesic, then its volume exceeds that of the Weeks manifold. Further, if the shortest geodesic of $M$ has length less than $\ell_{0}<0.1$, then its volume also exceeds that of the Weeks manifold. (The bounds obtained in [6] were $r_{0}>1.483$ and $\ell_{0}<0.069$.) Przeworski has further improved these bounds using packing arguments for congruent cylinders in hyperbolic space, [17.

We feel our approach here, while closely paralleling [6], is somewhat cleaner, sharper and offers greater hope for generalisation.

We remark that given the hugely computer intensive nature of the proposed search, it seems justifiable here to use a machine to make routine verifications such as establishing a function (often quite complicated) is increasing or decreasing, and so forth, rather than presenting pages of calculations. We have carried out all calculations on Mathematica and reported results to 3 decimals. This degree of accuracy

Received by the editors August 30, 2001 and, in revised form, April 10, 2003.

2000 Mathematics Subject Classification. Primary 30F40, 30D50, 57M50.

Research supported in part by the N. Z. Marsden Fund and the N. Z. Royal Society (James Cook Fellowship). 
in the situations under consideration only requires an elementary error analysis, indeed Taylor's error bounds are sufficient to guarantee the reported accuracy once we compute various derivatives (also performed by machine).

\section{Some Formulae}

Throughout we use the half-space model $\mathbb{H}^{3}$ of hyperbolic space. As usual, we identify the boundary points of $\mathbb{H}^{3}$ with the extended complex plane. For $x, y \in \mathbb{H}^{3}$, $\rho(x, y)$ will denote the hyperbolic distance between $x$ and $y$ and $I$ will denote the geodesic with endpoints 0 and $\infty$.

Given any two oriented geodesics $\gamma_{1}$ and $\gamma_{2}$ which do not meet either in $\mathbb{H}^{3}$ or on the boundary, we define uniquely $(\bmod 2 \pi)$ the rotation angle between them as follows. Let $\gamma$ be the common perpendicular to $\gamma_{1}$ and $\gamma_{2}$ oriented from $\gamma_{1}$ to $\gamma_{2}$ and let $r_{i}$ be the ray emanating along $\gamma_{i}$ from the point $\gamma_{i} \cap \gamma(i=1,2)$. Now define the rotation angle $\theta$ between $\gamma_{1}$ and $\gamma_{2}$ to be the angle obtained by going from $r_{1}$ to $r_{2}$ in the anticlockwise direction determined by the orientation of $\gamma$ and the right-hand rule. Clearly this definition is independent of the ordering of $\gamma_{1}$ and $\gamma_{2}$. If the geodesics are not oriented, then the rotation angle is still defined modulo $\pi$. Let $\delta\left(\gamma_{1}, \gamma_{2}\right)$ denote the complex distance between $\gamma_{1}$ and $\gamma_{2}$ (that is the complex number whose real and imaginary parts are respectively the distance and the rotation angle between $\gamma_{1}$ and $\gamma_{2}$ ). We abbreviate $\delta(\gamma, I)$ to $\delta(\gamma)$ (where $I$ is oriented from 0 to $\infty$ ).

For $z$ in the open right half-plane, $\mathbb{C}^{+}$, of $\mathbb{C}$ let $\alpha=\alpha(z)$ be the geodesic with endpoints $\tanh (z / 2)$ and $\operatorname{coth}(z / 2)$ oriented from $\tanh (z / 2)$ to $\operatorname{coth}(z / 2)$ so that, by [14] Lemma 5.2, we have that the geodesic $\alpha$ is the complex distance $z$ to $I$. Observe that adding $i \pi$ to $z$ just changes the orientation.

The geodesic $\alpha(z)$ can be considered as a canonical representative of all oriented geodesics with complex distance $z$ to $I$ and, if $\Re(\delta(\gamma))>0$, then $\gamma$ can be mapped to $\alpha(\delta(\gamma))$ by a complex dilation. We now define a mapping from these geodesics to $\mathbb{C}$. If $\gamma$ has (finite nonzero) endpoints $z_{1}$ and $z_{2}$ and is oriented from $z_{1}$ to $z_{2}$, let $c(\gamma)=z_{1} \sqrt{z_{2} / z_{1}}$, where we use the principal branch of the square root. (This is just a square root of $z_{1} z_{2}$, but not necessarily the principal branch.) Note that, for any complex $\lambda, c(\lambda \gamma)=\lambda c(\gamma)$ and that $c(\alpha(z))=1$. In geometric terms, $c(\gamma)$ is the point obtained by extending the perpendicular geodesic through $I$ and $\gamma$, in that order, to the boundary. This can be proved essentially by the argument of Lemma 5.3 [14]. However we do not need this information here.

For $t>0, z, w \in \mathbb{C}^{+}$, let

$$
\mathcal{A}(t, z, w)=\{\log (\lambda): \rho(\lambda \alpha(w), \alpha(z)) \leq t\}
$$

where the principal value of the logarithm is used.

Theorem 2.1. For $z, w \in \mathbb{C}^{+}, \lambda \in \mathbb{C}, \lambda \neq 0$, if $\delta(\alpha(z), \lambda \alpha(w))=v$, then

$$
\cosh (\log (\lambda))=\frac{\cosh (z) \cosh (w)-\cosh (v)}{\sinh (z) \sinh (w)}
$$


For each $z, w \in \mathbb{C}^{+}$and $0<t \leq \Re(z+w)$, let $\mathcal{E}(t, z, w)$ be the region bounded by the ellipse

$$
\begin{aligned}
& \left\{\frac{\cosh (z) \cosh (w)-\cosh (t+i \psi)}{\sinh (z) \sinh (w)}: 0 \leq \psi \leq 2 \pi\right\} \\
& \quad=\left\{\frac{\cosh (z \pm w)-\cosh (t+i \psi)}{\sinh (z) \sinh (w)} \mp 1: 0 \leq \psi \leq 2 \pi\right\} .
\end{aligned}
$$

The upper bound on $t$ (which we shall always assume tacitly) guarantees that -1 does not lie in the interior of $\mathcal{E}(t, z, w)$, hence, in this region, arccosh is defined unambiguously up to sign by taking taking \pm the principal branch. With this definition we have

\section{Corollary 2.1.}

$$
\mathcal{A}(t, z, w)=\operatorname{arccosh}(\mathcal{E}(t, z, w)) .
$$

Note that, when $t<\Re(z+w), \mathcal{A}(t, z, w)$ is bounded by a smooth curve (which double covers the ellipse $\mathcal{E}(t, z, w))$.

We defer the proof of Theorem 2.1 which is a direct, if lengthy, calculation. When $z=w$, Theorem 2.1 takes the following simpler form, which will be useful.

Corollary 2.2. Let $\lambda \in \mathbb{C}$ and suppose the complex distance between $\alpha(r+i \theta)$ and $\lambda \alpha(r+i \theta)$ is $t+i \psi$. Then

$$
\sinh \left(\frac{1}{2} \log \lambda\right)=\frac{i \sinh \left(\frac{t+i \psi}{2}\right)}{\sinh (r+i \theta)} .
$$

Clearly the set $\mathcal{A}(t, z, w)$ is centrally symmetric (that is $-\mathcal{A}(t, z, w)=\mathcal{A}(t, z, w)$ ). This region is not convex in general (for instance for $t$ small) and, although it will be convex in the cases of interest to us, we will in practice replace it by a geometrically simpler region, a disk or ellipse.

Let $\lambda \in \mathbb{C} \backslash\{0\}$ and let $\gamma_{1}, \gamma_{2}, \ldots, \gamma_{k}$ be geodesics with endpoints in $\mathbb{C} \backslash\{0\}$, and put

$$
\delta_{i}=\delta\left(\gamma_{i}\right)
$$

the associated spectrum of complex distances from $I$ and

$$
c_{i}=\log \left[c\left(\gamma_{i}\right)\right] .
$$

Let

$$
\begin{aligned}
\mathcal{P} & =\left\{\lambda^{n} \gamma_{i}: n \in \mathbb{Z}, 1 \leq i \leq k\right\} \\
\Lambda & =\{n \log (\lambda)+i 2 \pi m: n, m \in \mathbb{Z}\} .
\end{aligned}
$$

The pairwise distances between the geodesics in $\mathcal{P}$ exceeds $t$,

$$
\rho\left(\lambda^{n} \gamma_{i}, \lambda^{m} \gamma_{j}\right) \geq t
$$

if and only if for all $i, j$ the set

$$
c_{i}+\mathcal{A}\left(t, \delta_{i}, \delta_{j}\right)
$$

contains no point of $c_{j}+\Lambda$ except the point $c_{i}$ when $i=j$.

This effectively reduces to a statement about $k$ points on the torus $\mathbb{C} / \Lambda$ and the above requires (tacitly identifying points with their images under the quotient map)

$$
c_{j} \notin c_{i}+\mathcal{A}\left(t, \delta_{i}, \delta_{j}\right) \quad(i \neq j) .
$$


The condition (8) puts a lower bound on the distances between $c_{j}$ and $c_{j}$ which depend on $\delta_{i}, \delta_{j}$ and on $\arg \left(c_{i}-c_{j}\right)$. We will remove this dependence by replacing $\mathcal{A}\left(t, \delta_{i}, \delta_{j}\right)$ with a large convex centrally symmetric region $\Omega$, usually a disk or ellipse, in the intersection,

$$
\bigcap_{i, j} \mathcal{A}\left(t, \delta_{i}, \delta_{j}\right)
$$

The reason for doing this can be found in the following easy version of Minkowski's theorem.

\section{MinKowsKi's THEOREM}

Theorem 3.1. Let $\Lambda$ be a lattice in $\mathbb{R}^{n}$ of covolume $V$. Let $\mathcal{C}_{i j}(1 \leq i, j \leq k)$ and $\mathcal{C}_{i}(1 \leq i \leq k)$ be balanced subsets of $\mathbb{R}^{n}$ such that a packing of $\mathbb{R}^{n}$ by translates of the $\mathcal{C}_{i}$ has density at most $\rho$ and for all $i, j$,

$$
\frac{1}{2} \mathcal{C}_{i}+\frac{1}{2} \mathcal{C}_{j} \subseteq \mathcal{C}_{i j}
$$

Let $\Lambda_{i}, 1 \leq i \leq k$, be disjoint translates of $\Lambda$. Suppose that for all $i, j$, and each $x \in \Lambda_{i}$ the set $x+\mathcal{C}_{i j}$ contains no point of $\Lambda_{j}$ (other than $x$ itself if $i=j$ ). Then

$$
\sum_{i} \operatorname{Vol}\left(\mathcal{C}_{i}\right) \leq 2^{n} \rho V
$$

This estimate is sharp.

Proof. Suppose that in fact $\sum_{i} \operatorname{Vol}\left(\mathcal{C}_{i}\right)>2^{n} \rho V$. Then of course the set of translates $x_{i}+\frac{1}{2} \mathcal{C}_{i}$, where $x_{i} \in \Lambda_{i}$ cannot be disjoint. Hence there are $i, j$ and distinct $x_{i} \in \Lambda_{i}, x_{j} \in \Lambda_{j}$ for which

$$
\left(x_{i}+\frac{1}{2} \mathcal{C}_{i}\right) \cap\left(y_{j}+\frac{1}{2} \mathcal{C}_{j}\right) \neq \emptyset .
$$

Let $z \in\left(x_{i}+\frac{1}{2} \mathcal{C}_{i}\right) \cap\left(y_{j}+\frac{1}{2} \mathcal{C}_{j}\right)$. Then

$$
x_{i}-y_{j}=\left(x_{i}-z\right)-\left(y_{j}-z\right) \in \frac{1}{2} \mathcal{C}_{i}+\frac{1}{2} \mathcal{C}_{j} \subset \mathcal{C}_{i j} .
$$

That is, $y_{j} \in x_{i}+\mathcal{C}_{i j}$, contrary to our assumption. Sharpness is clear.

We next want to say a few general things about the set of distances between the geodesics forming the orbit of a given geodesic under a Kleinian group.

\section{The orthospectrum}

Let $\Gamma$ be a Kleinian group. Suppose that the map $z \mapsto \lambda z$ is a loxodromic element of $\Gamma$ whose axis, the hyperbolic line $\ell$ with endpoints 0 and $\infty$, is precisely invariant. That is, for each $g \in \Gamma$, either

$$
g(\ell)=\ell \quad \text { or } \quad g(\ell) \cap \ell=\emptyset,
$$

in the extended hyperbolic space. Let $\Gamma_{\ell}$ denote the stabiliser of $\ell$ in $\Gamma$. In general the group $\Gamma_{\ell}$ is virtually cyclic and generated by:

(1) a primitive loxodromic element $z \mapsto \mu z$,

(2) a primitive elliptic of order $n, z \mapsto e^{2 \pi i / n} z, n \geq 1$, and

(3) possibly an involution $z \mapsto \eta / z$, for some $\eta \in \mathbb{C} \backslash\{0\}$. 
If $\Gamma$ is torsion free, then cases (2) and (3) above do not occur. Every element $f \in \Gamma_{\ell}$ has the form

$$
f(z)=\mu^{j} e^{2 \pi i k / n} z \quad \text { or } \quad \mu^{j} e^{2 \pi i k / n} \eta / z
$$

for some pair of integers $j, k$, see [3].

If $\ell$ is precisely invariant, then the orbit of $\ell$ under the group $\Gamma$,

$$
\Gamma(\ell)=\{g(\ell): g \in \Gamma\},
$$

is partitioned into equivalence classes called orthoclasses. We say $\gamma, \gamma^{*} \in \Gamma(\ell)$ are conjugate if there is $g \in \Gamma$ such that

$$
g(\gamma)=\ell \text { and } g(\ell)=\gamma^{*} .
$$

Notice that if $\gamma_{1}^{*}, \gamma_{2}^{*}$ are both conjugates of $\gamma$, then there is $g \in \Gamma_{\ell}$ such that $g\left(\gamma_{1}^{*}\right)=\gamma_{2}^{*}$. An orthoclass $\mathcal{O}$ consists of a geodesic $\gamma \in \Gamma(\ell)$ together with any conjugate $\gamma^{*}$ and their orbits under $\Gamma_{\ell}$. That is,

$$
\mathcal{O}=\left\{g\left(\gamma \cup \gamma^{*}\right): g \in \Gamma(\ell)\right\} .
$$

Associated with an orthoclass $\mathcal{O}$ is the complex number $r_{\mathcal{O}}+i \theta_{\mathcal{O}}$ defined as the complex distance between any $\gamma_{i}$ in $\mathcal{O}$ and $\ell$. It is routine to check well-definedness; the only issue is with conjugates.

We order the orthoclasses as $\mathcal{O}(1), \mathcal{O}(2)$ etc., by the numbers $r_{\mathcal{O}}$. We ignore the trivial orthoclass with $r_{\mathcal{O}}=0$. The sequence of numbers $\left\{r_{\mathcal{O}(j)}\right\}_{j=1}^{\infty}$ is called the orthospectrum.

We now collect together a few facts we shall need about the orthospectrum. First, recall the following lemma from [10]; it is a generalisation of a well known result of Adams [1].

Lemma 4.1. Suppose that $\gamma, \gamma^{*} \in \mathcal{O}$ are conjugate. Then $\gamma^{*} \in \Gamma_{\ell}(\gamma)$ if and only if there is a primitive elliptic element $h$ of order two such that $h(\ell)=\gamma$. The distance between the axis of $h$ and the line $\ell$ is $r_{\mathcal{O}} / 2$.

A simple consequence of the definition is

Lemma 4.2. Let $\gamma_{1}, \gamma_{2} \in \mathcal{O}(1)$ and $\gamma_{1} \neq \gamma_{2}$. Then

$$
\rho\left(\gamma_{1}, \gamma_{2}\right)=r_{\mathcal{O}(1)} \quad \text { or } \quad \rho\left(\gamma_{1}, \gamma_{2}\right) \geq r_{\mathcal{O}(2)} .
$$

The next lemma is important. It shows that the first case of Lemma 4.2 can only occur in certain circumstances. It is a refinement of a result in [6]. It is very interesting to note with regard to related work (see [12]), the occurrence of Kleinian groups generated by elliptics of orders 2 and 3 in extremal situations again.

Lemma 4.3. Let $\Gamma$ be a Kleinian group and $\ell$ the axis of a loxodromic element of $\Gamma$, let $\mathcal{O}(i)$ be the ordered orthoclasses of $\Gamma(\ell)$ and let $f \in \Gamma$ such that $f(\alpha)=\ell$ for some $\alpha \in \mathcal{O}(1)$. Suppose $r_{\mathcal{O}(2)}>r_{\mathcal{O}(1)}$. Let $\gamma_{1}, \gamma_{2} \in \mathcal{O}(1)$. Then $\rho\left(\gamma_{1}, \gamma_{2}\right)=r_{\mathcal{O}(1)}$ if and only if the subgroup $\left\langle f, \Gamma_{\ell}\right\rangle$ contains elliptics of orders 2 and 3.

Indeed, there is $h \in \Gamma_{\ell}$ such that either $f h, f h f^{-1} h^{-1}$ or $f h f h^{-1}$ is elliptic of order 3.

Proof. Suppose that $\gamma_{1}, \gamma_{2} \in \mathcal{O}(1)$ and that $\rho\left(\gamma_{1}, \gamma_{2}\right)=r_{\mathcal{O}(1)}$. Let $f \in \Gamma$ such that $f\left(\gamma_{1}\right)=\ell$ and set $\gamma_{1}^{*}=f(\ell)$. Following [6] we consider two cases. 
Case 1. $\gamma_{2} \in \Gamma_{\ell}\left(\gamma_{1}\right)$. Then $\gamma_{2}=h_{1}\left(\gamma_{1}\right), h_{1} \in \Gamma_{\ell}$. As

$$
\rho\left(f\left(\gamma_{2}\right), \ell\right)=\rho\left(\gamma_{2}, f^{-1}(\ell)\right)=\rho\left(\gamma_{2}, \gamma_{1}\right)=r_{\mathcal{O}(1)}<r_{\mathcal{O}(2)},
$$

we must have $f\left(\gamma_{2}\right) \in \mathcal{O}(1)$. Thus $f\left(\gamma_{2}\right)$ is a translate of either $\gamma_{1}$ or $\gamma_{1}^{*}$ by an element of $\Gamma_{\ell}$. In the first case, $f\left(\gamma_{2}\right)=h_{2}\left(\gamma_{1}\right), h_{2} \in \Gamma_{\ell}$, whence $f h_{1} f^{-1}(\ell)=$ $h_{2} f^{-1}(\ell)$ and so there is $h_{3} \in \Gamma_{\ell}$ such that

$$
f h_{1} f^{-1}=h_{2} f^{-1} h_{3}, \quad h_{i} \in \Gamma_{\ell} .
$$

Similarly, if $f\left(\gamma_{2}\right)=h_{2}\left(\gamma_{1}^{*}\right)$, we again consider the orbit of $\ell$ to find

$$
f h_{1} f^{-1}=h_{2} f h_{3}, \quad h_{i} \in \Gamma_{\ell} .
$$

Case 2. $\gamma_{2} \in \Gamma_{\ell}\left(\gamma_{1}^{*}\right)$. Then $\gamma_{2}=h_{1}\left(\gamma_{1}^{*}\right), h_{1} \in \Gamma_{\ell}$. Then $h_{1} f\left(\gamma_{1}\right)=\ell$ and $h_{1} f(\ell)=$ $\gamma_{2}$. As before $\gamma_{3}=f^{-1} h_{1}^{-1}\left(\gamma_{1}\right) \in \mathcal{O}(1)$ as $\rho\left(\gamma_{3}, \ell\right)=\rho\left(\gamma_{1}, \ell\right)$. Then, as before, $\gamma_{3}$ is a translate of $\gamma_{1}$ or $\gamma_{1}^{*}$ by an element of $\Gamma(\ell) \cdot \gamma_{3}=h_{2}\left(\gamma_{1}\right)$ leads to the relation

$$
f^{-1} h_{1}^{-1} f^{-1}=h_{2} f^{-1} h_{3}, \quad h_{i} \in \Gamma_{\ell},
$$

while if $\gamma_{3}=h_{2}\left(\gamma_{1}^{*}\right)$, we obtain the relation

$$
f^{-1} h_{1}^{-1} f^{-1}=h_{2} f h_{3}, \quad h_{i} \in \Gamma_{\ell} .
$$

The relators in (14),(15), (16) and (17) all have a similar form. In (14) we put $g=f^{-1} h_{2}$ and rearrange to find $u g v=g^{2}, u, v \in \Gamma_{\ell}$. In (15) we put $g=h_{3} f$ to again find $u g v=g^{2}, u, v \in \Gamma_{\ell}$ (for different $u, v$ of course). In (16) we put $g=h_{1}^{-1} f^{-1}$ to once again get $u g v=g^{2}, u, v \in \Gamma_{\ell}$. Finally, in (17) we put $g=h_{1} f$ to find the slightly different relator $u g v=g^{-2}, u, v \in \Gamma_{\ell}$. In all four cases, the element $g \notin \Gamma_{\ell}$.

We next study the matrix equations corresponding to the relators above. The result we want follows from the next lemma which gives rather more precise information.

Lemma 4.4. Suppose that $\Gamma=\langle f, g, h\rangle$ is a Kleinan group, that $g$, $h$ stabilise a hyperbolic line $\ell$ and that $f(\ell) \cap \ell=\emptyset$, in the extended hyperbolic space. Suppose that for some choice of sign $\epsilon= \pm$ the equation,

$$
g f h=f^{\epsilon 2},
$$

holds in $\Gamma$; then $\Gamma$ is generated by elliptic elements of orders 2 and 3. Indeed:

- If neither $g$ nor $h$ is of order 2 , then $g=h, f$ is elliptic of order $3, f g$ is elliptic of order 2 , and $g f g=f^{2}$.

- If $h$ is of order 2 , then $g \mathrm{fg}^{-1} f^{-1}$ is elliptic of order 3 and $g^{-1} f$ has order 2 when $\epsilon=+$ and $g f g^{-1} f$ is elliptic of order 3 and fh has order 2 when $\epsilon=-$.

- If $g$ and $h$ are of order 2 , then $g=h$ and $f$ is elliptic of order 3 .

The proof we offer is found by studying all solutions of this matrix equation after appropriate normalisations are made (see $₫ 8$ ). 


\section{MANifold VOLUmeS}

In this section we give an elementary bound for the volume of a hyperbolic 3-manifold and develop a formula which we use to prove the main results of the paper. The volume estimate was, at the time of writing, best known. However a recent innovative approach has been found by Algol [2] which gives the lower bound 0.32. Computer experiments suggest that our arguments can be further refined to give better bounds than those we offer here. However the cost is in much greater expenditure of computer time and a much deeper error analysis to obtain rigour.

Theorem 5.1. Let $\Gamma$ be a torsion free Kleinian group and let $\ell$ be a precisely invariant loxodromic axis. Let $\log 3<r=r_{\mathcal{O}(1)}<4$ and $t=r_{\mathcal{O}(2)}<2 r$. Then

$$
\operatorname{Vol}\left(\mathbb{H}^{3} / \Gamma\right) \geq \mathcal{V}_{1}(r, t)=2 \sqrt{3} \sinh ^{2}\left(\frac{r}{2}\right) F(r, t)
$$

where

$$
F(r, t)=\operatorname{arcsinh}\left(\frac{\sinh (t / 2)}{\cosh (r)}\right) \sqrt{\operatorname{arcsinh}^{2}\left(\frac{\sinh (t / 2)}{\cosh (r)}\right)+\arcsin ^{2}\left(\frac{1}{\cosh (r)}\right)} .
$$

Proof. For each $r, \theta(z=r+i \theta)$ and $t$ in this range and following [10] we would like to find the ellipse of largest area, or more precisely a good approximation to such an ellipse, lying in the region enclosed by the curve

$$
\mathcal{E}(z, t)=\operatorname{arcsinh}\left(\frac{i \sinh (t / 2+i \psi)}{\sinh (r+i \theta)}\right), \quad 0<\psi<2 \pi .
$$

We can find a disk lying interior to this region. Namely, as

$$
\left|\frac{i \sinh (t / 2+i \psi)}{\sinh (r+i \theta)}\right| \geq \frac{\sinh (t / 2)}{|\sinh (r+i \theta)|}
$$

and as $|\sinh (w)| \leq \sinh |w|$, it is clear that the disk of radius

$$
s=\operatorname{arcsinh}\left(\frac{\sinh (t / 2)}{|\sinh (r+i \theta)|}\right) \geq \operatorname{arcsinh}\left(\frac{\sinh (t / 2)}{\cosh (r)}\right)
$$

whose center lies at the origin is such a disk.

Our interest is in those $r$ in the interval $[\log (3), 4]$ and there it is not difficult to see the above estimate is not particularly good and that much better ellipses can be found. However, obtaining an analytic proof for a formula escaped us, and so we took a computational approach. There is considerable numerical instability in dealing with functions like arcsinh and it is for this reason we adopted the following plan.

First, for a given $z=r+i \theta$ and $t$ we construct a candidate ellipse $E_{z, t}$ by guessing the axes and determining appropriate foci. Our guess for the minor axis will be the radius of the inscribed disk centered at the origin, the foci will lie on the line through the origin perpendicular to the points of tangency with this inscribed disk and the region $\mathcal{E}(z, t)$ and the major axis will be determined by what we consider to be the worst case. Preliminary computer experimentation suggests that given $r$ in the range of interest to us, the region containing the ellipse of smallest area is that where $\theta=\pi / 2$. Further experimentation suggests this ellipse has area at least

$$
A=4 \pi F(r, t) \text {. }
$$


All the ellipses we construct for given $r$ and $t$ will then have this area, up to our computational error which we shall bound rigorously. It is here that improvements can be made. For any given values of $\theta, r$ and $t$, it appears that ellipses of larger area exist. However, using these larger ellipses doesn't give enough slack for our elementary error analysis to work well.

Next, any set $E \subset \mathcal{E}(z, t)$ if for all $\zeta \in \partial E$,

$$
H(\zeta, z, t)=|\sinh (\zeta) \sinh (z)+1|+|\sinh (\zeta) \sinh (z)-1|-2 \cosh (t / 2)<0 .
$$

One can easily obtain Lipschitz bounds on $H(\zeta, z, t)$ using the inequality

$$
\begin{aligned}
\left|H(\zeta, z, t)-H\left(\zeta^{\prime}, z, t\right)\right| & \leq 2|\sinh (z)|\left|\sinh (\zeta)-\sinh \left(\zeta^{\prime}\right)\right| \\
& \leq 2|\sinh (z)||\cosh (\eta)|\left|\zeta-\zeta^{\prime}\right|
\end{aligned}
$$

for some point $\eta \in\left[\zeta, \zeta^{\prime}\right]$. Also we have, using first order Taylor series,

$$
\begin{aligned}
& \left|H(\zeta, z, t)-H\left(\zeta, z^{\prime}, t^{\prime}\right)\right| \\
& \quad \leq 2|\sinh (\zeta)|\left|\sinh (z)-\sinh \left(z^{\prime}\right)\right|+\sinh \left(t^{\prime \prime} / 2\right)\left|t-t^{\prime}\right| \\
& \quad \leq 2|\sinh (\zeta)|\left|\cosh \left(z^{\prime \prime}\right)\right|\left|z-z^{\prime}\right|+\sinh \left(t^{\prime \prime} / 2\right)\left|t-t^{\prime}\right|
\end{aligned}
$$

for some $z^{\prime \prime} \in\left[z, z^{\prime}\right]$ and $t^{\prime \prime} \in\left[t, t^{\prime}\right]$. Of course the inequality will persist if we choose $t^{\prime \prime}=\max \left\{t, t^{\prime}\right\}$ and $z^{\prime \prime}=\max \left\{\Re(z), \Re\left(z^{\prime}\right)\right\}$.

We must verify our formula in the range

$$
\log (3)<r<4, \quad 0 \leq \theta<\pi / 2 \quad \text { and } \quad r<t<2 r .
$$

The Lipschitz bounds at (24) are sufficient to give reliable estimates for a fine search of this region. To this end we choose $\delta>0$ and consider the box

$$
Q=Q_{\delta}(z, t)=[r-\delta, r] \times[\theta, \theta+\delta] \times[t-\delta, t] .
$$

Then we have for $\left(z^{\prime}, t^{\prime}\right) \in Q$,

$$
\left|H(\zeta, z, t)-H\left(\zeta, z^{\prime}, t^{\prime}\right)\right| \leq(2 \sqrt{2}|\sinh (\zeta)||\cosh (r+i \theta)|+\sinh (t / 2)) \delta .
$$

Therefore,

$$
H(\zeta, z, t)<-(2 \sqrt{2}|\sinh (\zeta)||\cosh (r+i \theta)|+\sinh (t / 2)) \delta
$$

implies that

$$
\zeta \in \mathbf{E}\left(z^{\prime}, t^{\prime}\right), \quad \text { for all }\left(z^{\prime}, t^{\prime}\right) \in Q .
$$

Hence, for a given $z, t$ and $\delta$, we seek to verify that (29) holds for all $\zeta \in E_{z, t}$. Then of course this ellipse works for all $\left(z^{\prime}, t^{\prime}\right) \in Q$. That is $E_{z, t} \subset \mathcal{E}\left(z^{\prime}, t^{\prime}\right)$. The inequality (29) is what we test numerically for a candidate ellipse. We now seek a good candidate.

5.1. A candidate ellipse. There are no computational issues in identifying a candidate ellipse, only in establishing its purported properties. We begin by finding the best inscribed disk. A pair of antipodal points on this disk at points of tangency with curve defining the region give us our candidate minor axis, whose normal should define the major axis. We seek to minimise the function $|f|^{2}$ where

$$
f(\psi)=\operatorname{arcsinh}\left(\frac{i \sinh (t / 2+i \psi)}{\sinh (r+i \theta)}\right) .
$$


The derivative with respect to $\psi$ is

$$
\frac{\partial|f|^{2}}{\partial \psi}=-2 \Re\left\{\frac{\cosh (t / 2+i \psi)}{\sqrt{\sinh ^{2}(r+i \theta)-\sinh ^{2}(t / 2+i \psi)}} \overline{f(\psi)}\right\} .
$$

We use the secant method to quickly identify a root $\psi_{0}$ of this equation in the interval $[0, \pi / 2]$. For values of $\theta$ away from $\pi / 2$ this root appears to be the minimum; near $\pi / 2$ we use the secant method to identify a root in $[\pi / 2, \pi]$ which appears to be the minimum in this case. This switch in roots is clear geometrically as these elliptical regions rotate. We now propose that the minor axis of a candidate ellipse is the line segment $\left[-f\left(\psi_{0}\right), f\left(\psi_{0}\right)\right]$. Set $a=f\left(\psi_{0}\right)$. An ellipse with this minor axis cannot satisfy (29) as it is, by construction, tangent to the region at the point $a$. To get (29) we must scale this ellipse down and therefore we multiply $a$ by a factor less than one, $a_{\epsilon}=(1-\epsilon) a$. The major axis is perpendicular to this axis. We choose the length to be

$$
|b|=\max \left\{A /\left(\pi\left|a_{\epsilon}\right|\right),\left|a_{\epsilon}\right|\right\}
$$

where $A$ is defined at (22). The proposed candidate ellipse is then parameterised by

$$
E_{z, t}=\left\{\zeta=\frac{a}{|a|}\left(\left|a_{\epsilon}\right| \cos (\psi)+i|b| \sin (\psi)\right): 0 \leq \psi<2 \pi\right\}
$$

whose area is clearly $A$. Now given our $\epsilon>0$ and $z=r+i \theta$ and $t$ we put

$$
\zeta_{\epsilon}(\psi)=\frac{a}{|a|}\left(\left|a_{\epsilon}\right| \cos (\psi)+i|b| \sin (\psi)\right) .
$$

5.2. Computation. The Lipschitz estimate (24) gives us

$$
\left|\frac{\partial}{\partial \psi} H\left(\zeta_{\epsilon}(\psi), z, t\right)\right| \leq 2|\sinh (z)|\left|\cosh \left(\zeta_{\epsilon}(\psi)\right)\right|\left|\zeta_{\epsilon}(\psi+\pi / 2)\right| .
$$

In our range of interest $\left|\zeta_{\epsilon}\right|<1$ and $\left|\zeta_{\epsilon}\right|$ decreases as $r$ increases. Ultimately we want our volume estimate to be accurate to three decimal places. We have set things up so that we expect this minimum to occur at $\psi=0$ (where the point of tangency with the unscaled ellipse is) and this makes determining the minimum computationally somewhat simpler using the derivative bounds at (34). The expected minimum is therefore the value $F((1-\epsilon) a, z, t)$, which from (29) gives us

$$
\delta=-F((1-\epsilon) a, z, t) /(2 \sqrt{2}|\sinh ((1-\epsilon) a)||\cosh (r+i \theta)|+\sinh (t / 2)) .
$$

All the variables controlling the numerical error have thus been determined. We implemented this search using a few lines of code in Mathematica. Thus

Lemma 5.1. The region $\mathcal{E}(r+i \theta, r+i \theta, t)$ contains an ellipse of area $4 \pi F(r, t)$.

The following lemma is routinely verified by machine.

Lemma 5.2. The function $2 \sqrt{3} \sinh ^{2}\left(\frac{r}{2}\right) F(r, t)$ is increasing in $r$ and $t$.

Next, choose $\alpha \in \mathcal{O}(1)$ and denote its conjugate by $\alpha^{*}$. The orbits of $\alpha$ and $\alpha^{*}$ under the stabiliser $\Gamma_{\ell}=\left\langle z \mapsto \lambda^{n} z\right\rangle$ are disjoint and, since $t \geq r$ and there are no elliptics of order 3 in $\Gamma$, no two distinct elements of these orbits are closer than the distance $t$. We may assume the point $c(\alpha)=1$ and set $c=\log \left(c\left(\alpha^{*}\right)\right)$. According to (17) the ellipse of the preceding corollary contains no points, other than the origin, 
of the lattices $\Lambda$ and $c+\Lambda$, where $\Lambda$ is defined at (5). Notice that if $\lambda=e^{\tau+i \psi}$, then

$$
\operatorname{Area}(\mathbb{C} / \Lambda)=2 \pi \tau
$$

Now Minkowski's theorem, Theorem 3.1 gives

$$
2 \pi \tau \geq 4 \sqrt{3} F(r, t)
$$

where we have used the fact that the optimal density of packings by congruent ellipses in the plane is $\pi / \sqrt{12}$. It follows that

$$
\pi \tau \sinh ^{2}\left(\frac{r}{2}\right) \geq 2 \sqrt{3} F(r, t) \sinh ^{2}\left(\frac{r}{2}\right) .
$$

The right-hand side of this equation represents the volume of the solid tube of length $\tau$ and radius $r$,

$$
\mathcal{C}(\ell, r) / \Gamma_{\ell}=\pi \tau \sinh ^{2}\left(\frac{r}{2}\right)
$$

where $\mathcal{C}(\ell, r)=\left\{x \in \mathbb{H}^{3}: \rho(x, \ell) \leq r\right\}$. The precise invariance of $\ell$ implies $\mathbb{H}^{3} / \Gamma \geq$ $\mathcal{C}(\ell, r) / \Gamma_{\ell}$. The result follows.

Corollary 5.1. A hyperbolic 3-manifold has volume at least 0.2855 .

Proof. Gabai, Meyerhoff and Thurston have classified all hyperbolic 3-manifolds which do not contain an embedded tubular neighbourhood of radius $(\log 3) / 2$ about their shortest geodesic [7]. Each such manifold has volume at least that of the Weeks manifold, and so we assume that the shortest geodesic has an embedded tubular neighbourhood of at least $r=(\log 3) / 2$. We set $t=r$. The lift of the shortest geodesic is a precisely invariant loxodromic axis $\ell$ for which we must have $r_{\mathcal{O}(1)}>\log 3$. We use the volume formula in the specified range of Theorem 5.1 For larger values of $r$ the easy estimate

$$
|\sinh (u)-\sinh (v)|+|\sinh (u)+\sinh (v)| \leq 2 \sinh \left(\frac{|u-v|+|u+v|}{2}\right)
$$

identifies an ellipse with foci $\pm v, v=\operatorname{arcsinh}\left(\frac{1}{\sinh (r+i \theta)}\right)$, which in turn gives good enough bounds. We leave the reader to develop the simple accompanying volume estimate. (We use this simple estimate later as well for larger values of $r$ and $t$.) The corollary now follows from the preceding theorem and lemma.

Following [6] we want to use the volume formula at (19) in the following way. If $r_{\mathcal{O}(1)}=r_{\mathcal{O}(2)}$, then we can essentially double the above estimate by choosing $\beta, \beta^{*} \in \mathcal{O}(2)$ and producing two more lattices which do not meet our inscribed ellipse. A little care is needed here since the complex distances might not be the same even though the real distances are. We set

$$
\Delta=r_{\mathcal{O}(2)}-r_{\mathcal{O}(1)}
$$

If $\Delta$ is small, then the above doubling argument needs to be slightly refined to produce a good result, while if $\Delta$ is large, that is $t \gg r$, then the above estimate is already good. We now turn to refine this volume estimate. We begin with the 
general formula of Theorem 2.1 We first observe

$$
\begin{aligned}
& \left|\frac{\cosh (r+i \theta) \cosh (s+i \phi)-\cosh (t+i \psi)}{\sinh (r+i \theta) \sinh (s+i \phi)}-1\right| \\
& \quad=\left|\frac{\cosh (r-s+i \theta-i \phi)-\cosh (t+i \psi)}{\sinh (r+i \theta) \sinh (s+i \phi)}\right| \\
& \quad \geq \frac{\cosh (t)-\cosh (r-s)}{\cosh (r) \cosh (s)}=\kappa,
\end{aligned}
$$

the last inequality being an exercise in calculus.

Hence the ellipse $\mathcal{E}(r+i \theta, s+i \phi, t)$ contains the disk $D(1, \kappa)$. We then have the next lemma.

Lemma 5.3. For all $0 \leq \theta, \phi \leq 2 \pi$, and $z=r+i \theta, w=s+i \phi$ and $t>0$ with $t>r-s$, the region $\mathcal{A}(t, z, w)$ contains a disk of radius

$$
\operatorname{arccosh}\left(1+\frac{\cosh (t)-\cosh (r-s)}{\cosh (r) \cosh (s)}\right)
$$

centered at the origin.

We apply this lemma as follows: we have $\alpha, \alpha^{*} \in \mathcal{O}(1)$ and so choose $\beta, \beta^{*} \in$ $\mathcal{O}(2)$. The orbits of these four geodesics are pairwise disjoint and no two are closer than the distance $r_{\mathcal{O}(1)}$. As before we assume that $c(\alpha)=1$ and set $c_{1}=\log \left(c\left(\alpha^{*}\right)\right)$, $c_{2}=\log (c(\beta))$ and $c_{3}=\log \left(c\left(\beta^{*}\right)\right)$. Now, as before, (7) shows us the disk of radius

$$
\kappa(\Delta)=\operatorname{arccosh}\left(1+\frac{\cosh \left(r_{\mathcal{O}(1)}\right)-\cosh (\Delta)}{\cosh \left(r_{\mathcal{O}(1)}\right) \cosh \left(r_{\mathcal{O}(1)}+\Delta\right)}\right)
$$

centered at one point of the four lattices $\Lambda, c_{1}+\Lambda, c_{2}+\Lambda$ and $c_{3}+\Lambda$ contains no other point of these four lattices. Minkowski's theorem, Theorem 3.1 gives

$$
2 \pi \tau \geq 2 \sqrt{3} \kappa^{2}(\Delta)
$$

using the optimal density estimate again. It follows that

$$
\pi \tau \sinh ^{2}\left(\frac{r}{2}\right) \geq \mathcal{V}_{2}(r, \Delta)=\sqrt{3} \kappa^{2}(\Delta) \sinh ^{2}\left(\frac{r}{2}\right) .
$$

We have proved the following theorem.

Theorem 5.2. Let $\Gamma$ be a torsion free Kleinian group and let $\ell$ be a precisely invariant loxodromic axis. Let $r=r_{\mathcal{O}(1)}$ and $t=r_{\mathcal{O}(2)}$. Then

$$
\operatorname{Vol}\left(\mathbb{H}^{3} / \Gamma\right) \geq \mathcal{V}_{2}(r, t-r) \text {. }
$$

As $\kappa^{2}(\Delta)$ is clearly decreasing in $\Delta$ this estimate get worse as $\Delta$ increases, while our previous estimate gets better.

The next theorem improves a result of [6]. It is obtained by balancing these two estimates of (19) and (43)).

Theorem 5.3. If $M$ is a hyperbolic 3-manifold that contains an embedded solid tube of radius $r_{0}=1.31$ about a closed geodesic, then $M$ has volume greater than that of the Weeks manifold.

Proof. We lift this closed geodesic and consider its orthospectrum. We have $r_{\mathcal{O}(1)}>$ $2.62=2 r_{0}$. Suppose $\Delta \leq 0.275$. Then Theorem 5.1 provides a volume bound of 0.943 , while if $\Delta \geq 0.275$ Theorem 5.2 provides a similar bound. Again, if $r_{\mathcal{O}(1)}>4$, we use the elementary volume estimates from the inscribed disk. 
Remark. The two noncompact orientable hyberbolic 3-manifolds of minimal volume have been identified as the Figure of 8 knot compliment and its sister by Cao-Meyerhoff, [5]. The above results have some relevance here. If the distance between elements of $\mathcal{O}(1)$ and $\mathcal{O}(2)$ exceeds $r_{\mathcal{O}(1)}$ (note it cannot be smaller than this number), then we have the improved estimate

$$
\kappa(\Delta) \geq \operatorname{arccosh}\left(1+\frac{\cosh \left(r_{\mathcal{O}(1)}+\Delta\right)-\cosh (\Delta)}{\cosh \left(r_{\mathcal{O}(1)}\right) \cosh \left(r_{\mathcal{O}(1)}+\Delta\right)}\right) .
$$

The associated volume estimate

$$
\sqrt{3} \kappa^{2}(\Delta) \sinh ^{2}\left(\frac{r_{\mathcal{O}(1)}}{2}\right)
$$

has the limit $\sqrt{3}$ independently of $\Delta$ as $r_{\mathcal{O}(1)} \rightarrow \infty$.

As soon as $\Delta \geq \log 2$ we also have $\mathcal{V}_{1}\left(r_{\mathcal{O}(1)}, r_{\mathcal{O}(1)}+\log (2)\right) \rightarrow \sqrt{3}$.

We recall from [14] that the optimal packing density of hyperbolic cylinders of radius $r$ cannot exceed $\left(1+23 e^{-r}\right) \rho_{\infty}$ where $\rho_{\infty}=0.853276 \ldots$ is the greatest possible density of a horoball packing in space. Our estimates above represent only the volume contributed by the cylinders about the orbit of the geodesic in question and therefore can be improved by this density factor. The limit volume (under the above restriction) is $\sqrt{3} / \rho_{\infty}=2.02 \ldots=V_{8}$, the volume of the Figure 8 knot compliment.

We were hoping that this method would provide an alternative route to (part of) the results of [5] by considering sequences of surgeries on the cusp of a candidate manifold, given that the sharp bound for the distances between the elliptic axes is known, [9, 6]. The estimates above would then imply that cylinders of radius $r_{\mathcal{O}(1)} / 2$ about elements of $\mathcal{O}(1)$ and $\mathcal{O}(2)$ are tangent which, in the limit, puts restrictions on the limit horoball packing. However these do not seem to simplify much of [5] and have the further disadvantage of not identifying the limit manifolds (just their volumes).

\section{Short GEOdESICS}

It is well known that sufficiently short geodesics in a hyperbolic 3-orbifold have an embedded tubular neighbourhood about them. This fact is a relatively straightforward consequence of Jørgensen's inequality [13] and good bounds were obtained by Meyerhoff in [15. Such estimates have since been sharpened and the following result is known, [11].

Theorem 6.1. Let $f \in \Gamma$, a Kleinian group, be a loxodromic element with translation length $\tau$. Then there is a precisely invariant collar about the axis $\ell$ of $f$ of radius $r$ where

$$
\sinh ^{2}(r) \geq \frac{\sqrt{3-4 \sqrt{3} \pi \tau}}{4 \pi \tau}-\frac{1}{2}
$$

Proof. Let $g \in \Gamma$ with $g(\ell) \neq \ell$ and $\delta=\rho(\ell, g(\ell))$. From [11] $\S 4$

$$
\sinh ^{2}(\delta / 2) \geq \frac{\sqrt{\left|\operatorname{tr}\left[g h g^{-1} h^{-1}\right]-2\right|}}{\left|t r^{2}(h)-4\right|}-\frac{1}{2}
$$

where $h$ is any element of $\Gamma_{\ell}$. Then [11] Lemma 4.7 implies there is $h \in \Gamma_{\ell}$ with

$$
\left|\operatorname{tr}^{2}(h)-4\right| \leq \frac{4 \pi \tau}{\sqrt{3}}
$$


Of course Jørgensen's inequality implies $\left|\operatorname{tr}\left[g h g^{-1} h^{-1}\right]-2\right|>1-\left|\operatorname{tr}^{2}(h)-4\right|$ which gives the general bound (46).

Next, recall that we in fact have two lower bounds on $\tau$ in terms of the first two elements of the orthospectrum. There are the bounds at (35) and (41). We are concerned only with the range $\log (3) \leq r_{\mathcal{O}(1)} \leq 2.62$. Below this range are the manifolds identified in [6] which have a shortest geodesic of length far exceeding 0.1 . While above this range we cannot have a minimal volume example. We compare both these bounds and find that the minimum, given the constraints (namely $\left.\mathcal{V}_{1}(r, t), \mathcal{V}_{2}(r, t-r)<0.943\right)$ exceeds 0.1 . We therefore obtain

Theorem 6.2. Let $\tau$ be the length of a shortest geodesic in a minimal volume hyperbolic 3-manifold. Then

$$
\tau>0.1
$$

\section{Proof of Theorem 2.1}

Recall the cross ratio of four complex numbers $[a, b, c, d]=\frac{(a-c)(b-d)}{(a-b)(c-d)}$. Let $v=$ $\delta(\alpha(z), \lambda \alpha(w))$ From [14] Lemma 5.2, or a direct computation, we have

$$
\begin{aligned}
-\sinh ^{2}(v / 2)= & {[\tanh (z / 2), \operatorname{coth}(z / 2), \lambda \tanh (w / 2) \lambda \operatorname{coth}(w / 2)] } \\
= & \frac{(\tanh (z / 2)-\lambda \tanh (w / 2))(\operatorname{coth}(z / 2)-\lambda \operatorname{coth}(w / 2))}{\lambda(\tanh (z / 2)-\operatorname{coth}(z / 2))(\tanh (w / 2)-\operatorname{coth}(w / 2))} \\
= & \lambda^{-1}(\cosh (w / 2) \sinh (z / 2)-\lambda \sinh (w / 2) \cosh (z / 2)) \\
& (\cosh (z / 2) \sinh (w / 2)-\lambda \sinh (z / 2) \cosh (w / 2)) .
\end{aligned}
$$

Let us put

We obtain the quadratic for $\lambda$,

$$
a=\sinh ^{2}\left(\frac{r+i \theta}{2}\right), \quad b=\sinh ^{2}\left(\frac{s+i \phi}{2}\right) .
$$

$$
1+\lambda \frac{(v-b(a+1)-a(b+1))}{\sqrt{b(a+1) a(b+1)}}+\lambda^{2}=0 .
$$

Then, since the roots are reciprocals,

$$
\lambda+\frac{1}{\lambda}=-\frac{(v-b(a+1)-a(b+1))}{\sqrt{b(a+1) a(b+1)}} .
$$

Notice that $4 a(a+1)=4 \sinh ^{2}(z / 2) \cosh ^{2}(z / 2)=\sinh ^{2}(z)$ and similarly $4 b(b+1)=$ $\sinh ^{2}(w)$. While

$$
\begin{aligned}
a(b+ & 1)+b(a+1) \\
= & \sinh ^{2}(z / 2) \cosh ^{2}(w / 2)+\cosh ^{2}(z / 2) \sinh ^{2}(w / 2) \\
= & \frac{1}{2}(\cosh (z) \cosh (w)-1) .
\end{aligned}
$$

This, together with the above, gives

$$
\frac{1}{2}\left(\lambda+\frac{1}{\lambda}\right)=\frac{\cosh (z) \cosh (w)-\cosh (v)}{\sinh (z) \sinh (w)}
$$

as desired. 


\section{Proof of Lemma 4.4}

Proof. The proof consists of considering the solutions to the corresponding matrix equation in $S L(2, \mathbb{C})$. We may assume that $g$ and $h$ stabilise the line with endpoints 0 and $\infty$. We put

$$
A=\left(\begin{array}{cc}
a & 0 \\
0 & 1 / a
\end{array}\right), \quad B=\left(\begin{array}{cc}
b & 0 \\
0 & 1 / b
\end{array}\right), \quad C=\left(\begin{array}{cc}
0 & c \\
-1 / c & 0
\end{array}\right), \quad D=\left(\begin{array}{cc}
0 & d \\
-1 / d & 0
\end{array}\right)
$$

and

$$
X=\left(\begin{array}{cc}
x & 1 \\
x y-1 & y
\end{array}\right)
$$

Representatives for $g$ and $h$ may be chosen from $A, B, C, D$ while the representative for $f$ is $X$. Various symmetries reduce us to considering the six cases

$$
A X B=X^{ \pm 2}, \quad A X C=X^{ \pm 2}, \quad C X D=X^{ \pm 2}
$$

We choose a couple of representative cases, the remainder are left for the reader to verify - a straightforward calculation using a computational algebra package such as Mathematica. We first compute

$$
\begin{gathered}
Y=A X B=\left(\begin{array}{cc}
a b x & a / b \\
(x y-1) b / a & y /(a b)
\end{array}\right) \\
Z=X^{2}=\left(\begin{array}{cc}
x(x+y)-1 & x+y \\
(x+y)(x y-1) & y(x+y)-1
\end{array}\right) .
\end{gathered}
$$

The two equations $Y_{11}=Z_{11}$ and $Y_{12}=Z_{12}$ imply that

$$
y=\frac{a}{b}+\frac{b}{a\left(b^{2}-1\right)}, \quad x=\frac{b}{a\left(1-b^{2}\right)}
$$

which we substitute in. Then the equation $Y_{21}=Z_{21}$ gives the possible solutions $b=0, b= \pm a$ and $b= \pm \sqrt{\left(a^{2}-1\right) / a^{2}}$. Of course $b=0$ is not allowed. If $b= \pm \sqrt{\left(a^{2}-1\right) / a^{2}}$, then we compute that $X_{21}=0$ so $f(\infty)=\infty$ and both $f$ and $g$ share a fixed point on the boundary. This is not possible in a discrete group, [3]. This leaves $b= \pm a$. In that case we compute

$$
X=\left(\begin{array}{cc}
1 /\left(a^{2}-1\right) & 1 \\
& -a^{2} /\left(a^{2}-1\right)
\end{array}\right), \quad X A=\left(\begin{array}{cc}
a /\left(a^{2}-1\right) & 1 / a \\
& -a /\left(a^{2}-1\right)
\end{array}\right),
$$

so that $f$ is elliptic of order $3, g=h$ and $f g$ has order 2 .

The remaining cases proceed in an entirely similar fashion.

\section{REFERENCES}

[1] C. Adams, The noncompact hyperbolic 3-manifold of minimal volume, Proc. Amer. Math. Soc., 100 (1987), 601-606. MR 88m:57018

[2] I. Algol, Volume change under drilling, to appear.

[3] A. Beardon, The geometry of discrete groups, Springer-Verlag, 1981.

[4] C. Cao, F.W. Gehring and G.J. Martin, Lattice constants and a lemma of Zagier, Lipa's legacy (New York, 1995), 107-120, Contemp. Math., 211, Amer. Math. Soc., Providence, RI, 1997. MR 99a:30040

[5] C. Cao and R. Meyerhoff, The orientable cusped hyperbolic 3-manifolds of minimum volume, Invent. Math., 146 (2001), no. 3, 451-478. MR 2002i:57016]

[6] D. Gabai, R. Meyerhoff and P. Milley, Volumes of tubes in hyperbolic 3-manifolds, J. Differential Geom., 57 (2001), no. 1, 23-46. MR 2002i:57017

[7] D. Gabai, R. Meyerhoff and N. Thurston, Homotopy hyperbolic 3-manifolds are hyperbolic, Annals of Math., to appear. 
[8] F. W. Gehring, T. H. Marshall and G.J. Martin, On the spectrum of axial distances in Kleinian groups, Indiana Math. J., 47 (1998), 1-10. MR 2000b:30066

[9] F. W. Gehring and G. J. Martin, Commutators, collars and the geometry of Möbius groups, J. Anal. Math., 63 (1994), 175-219. MR 96c:30040

[10] F. W. Gehring and G. J. Martin, Precisely invariant collars and the volume of hyperbolic 3-folds. J. Differential Geom., 49 (1998), no. 3, 411-435. MR 2000c:57030

[11] F. W. Gehring and G. J. Martin, The volume of hyperbolic 3-folds with $p$-torsion, $p \geq 6$. Quart. J. Math. Oxford Ser., 50 (1999), no. 197, 1-12. MR 2000c:57031

[12] F. W. Gehring, C. Maclachlan G. J. Martin and A. W. Reid, Arithmeticity, Discreteness and Volume, Trans. Amer. Math. Soc., 349 (1997), 3611-3643. MR 98d:57022

[13] T. Jørgensen, On discrete groups of Möbius transformations, Amer. J. Math., 78 (1976), 739-749. MR 55:658

[14] T. H. Marshall and G. J. Martin, Cylinder packings in hyperbolic space, preprint.

[15] R. Meyerhoff, A lower bound for the volume of hyperbolic 3-manifolds Canadian J. Math., 39 (1987), 1038-1056. MR 88k:57049

[16] A. Przeworski, Tubes in hyperbolic 3-manifolds, Thesis. University of Chicago and Top. and Appl. 128/2-3, 103-122.

[17] A. Przeworski, Density of tube packings in hyperbolic space, to appear.

Department of Mathematics, University of Auckland, Auckland, New Zealand

E-mail address: t_marshall@math.auckland.ac.nz

Department of Mathematics, University of Auckland, Auckland, New Zealand

E-mail address: martin@math.auckland.ac.nz 